\title{
Anabases
}

ANABASESS Traditions et réceptions de l'Antiquité

19 | 2014

Varia

\section{Les références aux textes « littéraires » dans Démocratie antique et démocratie moderne de Moses Finley}

Didier Pralon

\section{(2) OpenEdition Journals}

Édition électronique

URL : http://journals.openedition.org/anabases/4614

DOI : 10.4000/anabases.4614

ISSN : 2256-9421

Éditeur

E.R.A.S.M.E.

\section{Édition imprimée}

Date de publication : 1 avril 2014

Pagination : 97-109

ISSN : $1774-4296$

\section{Référence électronique}

Didier Pralon, « Les références aux textes « littéraires » dans Démocratie antique et démocratie moderne de Moses Finley », Anabases [En ligne], 19 | 2014, mis en ligne le 01 avril 2017, consulté le 10 décembre 2020. URL : http://journals.openedition.org/anabases/4614 ; DOI : https://doi.org/10.4000/ anabases.4614 
Anabases 19 (2014), p. 97-109

\section{Les références \\ aux textes " littéraires" \\ dans Démocratie antique \\ et démocratie moderne \\ de Moses Finley}

Didier PRALON

Moses Finley, fondamentalement un historien, a, par le truchement de Pierre Vidal-Naquet, appris aux étudiants français de ma génération, même aux philologues, à lire les textes grecs dans une perspective historique renouvelée. Je ne reviendrai pas sur la surprise et même la découverte qu'a été pour nous la lecture du Monde d'Ulysse ${ }^{1}$. Je pourrais ajouter Les premiers temps de la Grèce ${ }^{2}$, qui retraçaient et théorisaient les âges les plus obscurs de l'histoire grecque de 4000 à 479 avant notre ère... et y ajouter finalement l'ensemble de ses textes que nous avons lus au fil de leurs publications.

Quand André Tchernia a voulu m'embarquer dans cette célébration française du centenaire de Moses Finley, sans doute s'est-il souvenu du colloque que nous avions organisé avec Christian Goudineau, en 1976, à Aix-en-Provence, autour de l'Économie antique $^{3}$. La forme en était une suite de dialogues autour des questions que suscitait l'ouvrage : les particularités de l'économie antique, les statuts sociaux, le couple

1 The World of Odysseus, New York, The Viking Press, 1954, revu en 1976 ; traduction française par Claude Vernant-Blanc et Monique Alexandre, Paris, Maspéro, 1969 ; traduction de l'édition revue, 1978, réédition, Paris, La Découverte, 1986.

2 Early Greece: The Bronze and Archaic Ages, London, Chatto \& Windus ; New York, Norton, 1970, 2e édition, 1981, traduit par François Hartog, Paris, Maspero, 1973.

3 M.I. Finley, The Ancient Economy, Berkeley and Los Angeles, 1973; L'Économie antique, traduit par M.P. Higgs, Paris, Minuit, 1975. 
maîtres-esclaves, le monde paysan, le rapport des villes et de leurs campagnes, le rôle de l'État dans l'économie, le tout se développant dans les méandres de la longue durée et de la dualité Orient-Occident, ou, pour le dire plus crûment, Grèce-Rome.

Je travaillais alors sur la démocratie athénienne, j’ai donc proposé une lecture " naïve " de Démocratie antique et démocratie moderne ${ }^{4}$, ou, si l'on veut, une réflexion sur l'usage que fait Moses Finley des textes littéraires classiques. Par choix méthodologique autant que personnel, ma communication portera sur les citations, les évocations ou les analyses de textes littéraires grecs dans Démocratie antique et démocratie moderne. J'aurais préféré examiner les articles d'On a perdu la guerre de Troie ${ }^{5}$, dont je recommande particulièrement l'article assassin sur le malheureux Pindare, qui prouve que Moses Finley était allergique à la poésie hermétique; mais il aurait été vain de faire double emploi avec Évelyne Scheid.

Avant toute chose, un jugement esthétique : Moses Finley, homme de conviction et d'humour écrit d'une plume percutante. C'est encore aujourd'hui un plaisir de le lire ou de le relire.

Les trois conférences de Démocratie antique et démocratie moderne, prononcées en 1972 à l'université Rutgers, d'où Moses Finley avait été exclu en 1952 par la volonté de la commission McCarthy, développent une réflexion sur les théories modernes de la démocratie. Les références à la démocratie antique ne viennent qu'en appui et en miroir de la réflexion politologique : l'ordre des mots du titre aurait pu être inversé ${ }^{6}$ ! Il n'y est pas question, comme dans Esclavage antique et idéologie moderne ${ }^{7}$, de montrer

4 Democracy Ancient and Modern, Rutgers University Press ; London, Chatto \& Windus, 1973. Démocratie antique et démocratie moderne, traduction française de Monique Alexandre, Paris, Payot, 1976.

5 Aspects of Antiquity : Discoveries and Controversies, London, Chatto \& Windus, 1968, traduction de Jeannie Carlier, Paris, Les Belles Lettres, 1990.

6 Je conseille de méditer l'avant-propos de P. Vidal-Naquet à Démocratie antique et démocratie moderne: "Tradition de la démocratie grecque " p. 7-44. Dans sa préface à L'Invention de la politique, "Finley, les anciens et les modernes ", Paris, Flammarion, 1985, p. 5-15 (le titre de l'édition anglaise est plus pertinent: Politics in the Ancient World, Cambridge, 1985), il souligne le contraste entre les deux ouvrages : "Il s'agissait de contester sérieusement, parce qu’il s'agit de choses sérieuses, l'idée que la démocratie moderne ne fonctionne jamais aussi bien que lorsque les citoyens sont passifs. Cette théorie avait été ébranlée par le grand mouvement social et politique des années soixante, mouvement par lequel la société tentait plus ou moins de s'autoinstituer. Finley la mettait à mal en rappelant l'existence d'une démocratie où l'activisme n'était pas considéré comme un mal mais comme un bien, Athènes... Dans L'Invention de la politique, l'accent est mis non sur notre monde mais sur le monde antique" (p. 10).

7 Ancient Slavery and Modern Ideology, London, Chatto \& Windus, 1979, New York, The Viking press, 1980, traduit par D. Fourgous, Paris, Minuit, 1981. Dans sa préface, Finley explicite son projet ; il veut montrer « comment naquirent les sociétés esclavagistes de l'Antiquité et comment elles se transformèrent dans le long processus qui aboutit au féodalisme médiéval; comment fonctionna l'esclavage dans le cadre de l'économie antique 
les approximations idéologisées, voire les erreurs, des interprétations modernes et les différences entre l'esclavage antique et les esclavages modernes ; ni de tenter, comme dans L'Économie antique, une synthèse des singularités économiques anciennes, sans pour autant négliger les variations et les évolutions : "Bien que je me préoccupe de façon constante dans ce livre des changements et des variations et qu'on y trouve de nombreuses indications chronologiques, ce n'est pas ce qu'on appellerait une "histoire économique" ", écrit-il, p. 7 de la traduction française. On pourrait citer bien d'autres exemples de ce va et vient perpétuel et polymorphe entre l'Antiquité et les temps actuels, par exemple dans The Use and Abuse of History ${ }^{8}$, mais ceci devrait faire l'objet d'une étude spécifique.

Le corpus des textes antiques auxquels Moses Finley renvoie dans Démocratie antique et démocratie moderne, ou sur lesquels il s'appuie est somme toute assez limité (22 textes au total). Peu d'auteurs y figurent : l'Iliade d'Homère, la Constitution des Athéniens du vieil oligarque transmise parmi les œuvres de Xénophon, la Guerre du Péloponnèse de Thucydide, les Acharniens, les Nuées, la Lysistrata et les Thesmophories d'Aristophane, le Protagoras, le Ménon, le Gorgias, la République et les Lois de Platon, l'Apologie de Socrate de Xénophon, la Politique et la Constitution d'Athènes d'Aristote. Comme ces textes se justifient par le contexte des réflexions de Finley, je les examinerai dans l'ordre où ils apparaissent dans l'ouvrage.

\section{Chapitre 1. Dirigeants et dirigés (p. 47-90) ${ }^{9}$}

P. 48, une phrase de S.M. Lipset ${ }^{10}$ à propos de l'attirance exercée par les mouvements extrémistes sur les déclassés évoque à Finley les objections de Platon contre le rôle des travailleurs manuels ( $\beta \alpha ́ v \alpha v \sigma o ı$, sans référence) ${ }^{11}$ et l'affirmation d'Aristote (Politique VI 1319a19-38), selon laquelle les meilleures démocraties supposent un

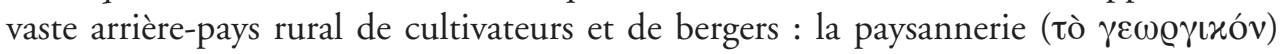
se contente de peu, est dure à la peine et peu exigeante, alors que le citadin de basse

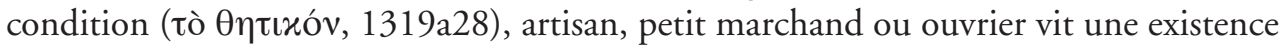

et des systèmes politiques anciens et comment on le jugea socialement et moralement ; ce qu'ont fait les historiens modernes de l'esclavage antique et pourquoi... L'esclavage moderne touchait des Noirs ; il est donc impossible d'en discuter sérieusement sans buter sur les tensions sociales et raciales actuelles."

8 The Use and Abuse of History, London, Chatto \& Windus, New York, The Viking Press, 1975. Plusieurs articles du recueil ont été traduits en français dans Mythe, mémoire et histoire, Paris, Flammarion, 1981, le premier donnant son titre au recueil français.

9 Les références à Démocratie antique et démocratie moderne renvoient à la traduction française (1976).

10 S.M. Lipset, Political man, New York, Garden City, 1960, p. 178, traduit par G \& G. Durand, L'homme et la politique, Paris, Seuil, 1963, p. 194.

11 On trouve 13 occurrences de ßávavoos dans le corpus platonicien. 
vile ( $\beta$ íos $\phi \alpha \tilde{v} \lambda$ os, 1319a25) et se regroupe facilement. Moses Finley suggère qu'une sociologie politique trop simple dissimule une idéologie antidémocratique. En ce sens, le langage édulcoré d'Aristote ne trompe guère : "Les cultivateurs, parce qu'ils sont dispersés sur le territoire, ni ne se rencontrent ni n'ont semblablement (aux citadins) besoin de ce genre de réunion "celles de l'agora” "(1319a30-32). Aux yeux d'Aristote, bon disciple en cela de Platon, la meilleure démocratie serait celle où le peuple ne se réunit pas ou aussi peu que possible ! Finley ne pouvait laisser passer le trait d'humour que l'on n'ose croire volontaire. Il n'y insiste pas.

P. 59, un autre trait d'humour (involontaire ?) est relevé chez Aristote, Politique III 1279b34-1280a4, à l'appui de l'ambiguïté du terme $\delta \tilde{\eta} \mu \mathrm{s}$ (" peuple dans son ensemble» ou « petit peuple») : «Le raisonnement paraît rendre évident que la souveraineté du petit nombre ou de la multitude n'est qu'accidentelle, l'une dans les oligarchies, l'autre dans les démocraties, du fait que les gens aisés sont peu nombreux, alors que les démunis sont partout nombreux (c'est pourquoi il n'arrive pas que les causes exprimées soient celles de la différence) ; la différence qui sépare la démocratie et l'oligarchie l'une de l'autre, c'est la pauvreté et la richesse et nécessairement là où les gouvernements gouvernent à cause de la richesse, qu'ils soient moins ou plus nombreux, c'est une oligarchie, là où gouvernent les démunis, c'est la démocratie (ma traduction). » Tant de cynisme candide étonne, mais n'a pas besoin d'explication. Dans son ingénuité lucide, Aristote débusque le non-dit, la raison dissimulée. Elle conforte l'évidence sous-jacente d'un lieu commun tenace des adversaires de la démocratie authentique.

P. 63, hors de son contexte, la phrase connue d'Aristote : "devenir maîtres de ceux qui méritent d'être réduits en esclavage » (Politique VII 1334a2), apparaît moins brutale encore qu'elle ne l'est en situation. Il faut, comme y invite la référence complexe de Finley, citer toute la phrase : "La pratique des arts de la guerre, on ne doit pas s'y adonner pour asservir ceux qui n'ont pas de dignité, mais d'abord pour soi-même, pour ne pas être asservi à d'autres, ensuite de manière à rechercher l'hégémonie dans l'intérêt de ceux qui sont gouvernés, et non pour être les maîtres de tous ; troisièmement en sorte d'être maîtres de ceux qui méritent d'être esclaves " (Politique VII 1333b38-1333a2). La première raison, simpliste, de combattre c'est de se défendre soi-même (sans considération d'obligation patriotique !) ; la seconde se fonde sur le faux-semblant ou l'illusion, comme on voudra, d'une sollicitude envers ceux que l'on gouverne, y compris ceux que l'on vainc et soumet (quel impérialisme ultérieur a trouvé une meilleure formule pour justifier l'appétence d'hégémonie ?) ; ne vient qu'en troisième lieu la domination sur ceux qui méritent d'être esclaves (sous la forme d'un

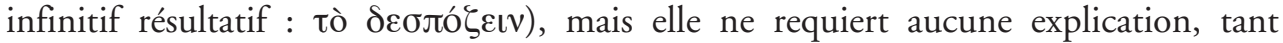
il paraît évident que certains seraient naturellement destinés à être esclaves et qu'il n'y aurait aucune raison de laisser autrui profiter de l'aubaine qu'ils représentent : un tel franc-parler déconcerte, du plus trivial au plus insupportable.

P. 65, est évoquée la question de la taille que doit avoir la cité idéale (Aristote, Politique VII 1326b3-7) : trop petite, elle ne peut être autarcique ; " composée d'un très grand nombre de gens, elle est autarcique dans l'ordre des nécessités, comme un peuple, 
mais pas en tant que cité : il n'est pas facile qu'y existe une vie politique ; qui en effet, y sera stratège d'une masse par trop excessive; qui en sera le héraut s'il n'est pas digne de Stentor ? " Le préconisateur du juste milieu prône, comme il se doit, une cité d'une taille modérée, favorable à une politique du "face à face ». On ne peut manquer de soupçonner dans la formulation une pointe contre les ambitions et les pratiques expansionnistes d'Alexandre, le disciple oublieux des leçons de Miéza. On sait combien longtemps le modèle grec a servi à récuser la possibilité d'un état démocratique vaste et populeux.

P. 68, en passant, Moses Finley relève, référence à l'appui, que, pour Aristote, "les élections sont aristocratiques et non démocratiques " parce qu' " elles introduisent un élément de choix délibéré, de sélection des meilleurs citoyens, les aristoi, au lieu du gouvernement par le peuple tout entier» (Politique IV 1300b4-5). Se dégage du contexte une taxinomie complexe des manières de désigner les magistrats (1299b11300b5). Y sont distingués les quatre modes de désignation des magistrats dans les quatre régimes susceptibles de délégation (la monarchie, elle, excluant implicitement toute représentation du peuple) : les deux purs (l'" aristocratique » et le " politique proprement $\left.\operatorname{dit}^{12} »\right)$, les deux impurs (l'« oligarchique » et le « démotique »/ « démocratique »). Ce n'est cependant pas cela qui intéresse Finley. Ce qu'il retient, c'est le constat brut, sans analyse ni explication, néanmoins pertinent, de la dérive aristocratique de tout mode de délégation par désignation.

P. 69/70, Finley, notant au passage que « la plupart des Athéniens ne dépassaient guère le niveau d'une "demi instruction" ", allègue Platon, sans référence précise, et cite

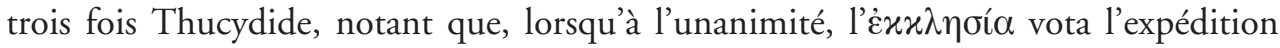
de Sicile en 415, " la plupart des Athéniens étaient généralement mal renseignés sur l'étendue de ce pays et sur le nombre de ses habitants" (Thucydide VI 1, 1). À cela les adversaires de la démocratie pourraient ajouter la suite immédiate de la remarque : « et ils ne se rendaient pas compte qu'ils suscitaient une guerre pas de beaucoup inférieure à la guerre contre les Péloponnésiens ". La preuve n'admet pas de réplique, sauf à soupçonner Thucydide de mauvaise foi, plus encore que de malveillance, alors qu'il admet que l'expédition fut soigneusement préparée (Thucydide VI 31, sans citation). Finley ne résiste pas à l'envie de citer l'une de ces délicieuses esquisses thucydidéennes, celle dans laquelle sont décrites les attentes de la plupart des citoyens et, en contraste, la réserve timorée des opposants (p. 70-71) : "Tout le monde, sans distinction, se passionna pour l'entreprise. Les gens âgés espéraient qu'on ferait la conquête de cette terre qu'on allait attaquer, ou du moins qu'une force aussi considérable serait à l'abri des accidents. Quant aux hommes en âge de porter les armes, ils aspiraient à voir du

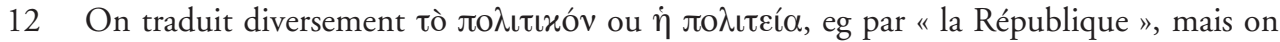
en altère le sens précis : le terme désigne le régime mixte du juste milieu, entre oligarchie et démocratie, dans lequel les institutions de la démocratie sont tempérées par l'existence d'un cens modéré (Politique IV 1293b30-38). C'est, selon Aristote, le régime modéré, le plus correct, par conséquent celui qui mérite le mieux le nom de " politique ". 
pays et à voir cette contrée lointaine, dont ils comptaient bien revenir sains et saufs. La masse des petites gens appelés à servir pensaient à l'argent qu'ils allaient dès à présent gagner et aux conquêtes futures grâce auxquelles ils pourraient devenir les salariés perpétuels de l'État. L'enthousiasme de la majorité était tel que ceux qui désapprouvaient l'entreprise, craignant de passer pour de mauvais patriotes s'ils votaient contre elle, évitaient de se manifester " (Thucydide, Guerre du Péloponnèse, VI 24, 3-25, 1, traduction de D. Roussel, 1964). Mais Finley prévient aussitôt le risque d'imaginer là une foucade irréfléchie (p. 71) : "Ce serait une erreur de ne pas tenir compte de ce que le vote de l'Assemblée d'envahir la Sicile avait été précédé d'une période de discussions intenses dans les boutiques et les tavernes, sur la place de la ville, lors des dîners - des discussions qui se déroulaient entre ces mêmes hommes qui finalement se réunirent sur la Pnyx pour les débats et le vote officiel. " Un second tableau, lui aussi évocateur, nuance celui de Thucydide.

P. 73, pour étayer l'idée que la démocratie athénienne fonctionnait plutôt bien, Finley cite le témoignage, tout à la fois réaliste et ironique, d'un ennemi déterminé de cette démocratie, le vieil oligarque, appelé traditionnellement le pseudo-Xénophon, auteur d'une Constitution des Athéniens $(3,1)$ : "Quant au système de gouvernement athénien, je ne l'aime pas. Cependant, depuis que les Athéniens ont décidé de devenir une démocratie, il me semble qu'ils maintiennent bien la démocratie " (traduction sans doute de Monique Alexandre, du texte de Finley). Ici encore, la preuve ne souffre aucune réserve, ne suscite aucun commentaire, si ce n'est que le vieil oligarque n'est pas totalement aveuglé par ses préventions, mais n'en n'admet pas pour autant que la démocratie est un régime estimable ; il lui reconnaît seulement d'être viable.

P. 73, immédiatement après avoir cité le vieil oligarque, Finley relevant une contradiction entre un propos affichant la participation potentielle de tous au fonctionnement de la démocratie et la pratique effective, cite « l'oraison funèbre » de Périclès : "Quand un homme sans fortune peut rendre quelque service à l'État, l'obscurité de sa condition ne constitue pas pour lui un obstacle " (Thucydide, Guerre du Péloponnèse II 37, 1, trad. D. Roussel). La remarque s'inscrit dans un développement sur l'égalité ( ò ĺoov) en démocratie.

P. 75, Pour récuser l'idée que, dans l'Athènes démocratique, les dirigeants auraient pu décider plutôt que le peuple, Finley cite de nouveau Thucydide (Guerre du Péloponnèse I 140,1) et, plus précisément, le discours de Périclès au début du conflit : "Je vois qu'en cette occasion, je dois vous donner exactement le même avis que je vous ai donné dans le passé ; et je demande à ceux d'entre vous qui sont persuadés, de donner leur appui à ces résolutions que nous sommes en train de prendre tous ensemble ». La traduction de Finley me paraît plutôt une paraphrase. Est omise la traduc-

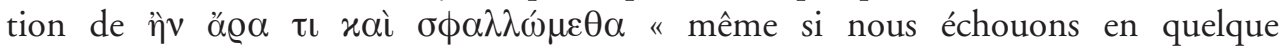

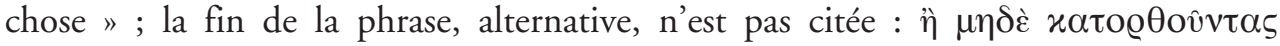

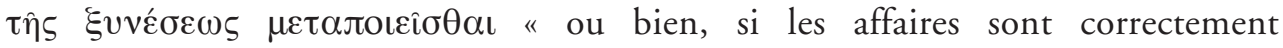
menées, de ne pas en revendiquer l'intelligence ». Elle rappelle bien pourtant a contrario la responsabilité réelle du peuple dans les prises de décision. 
P. 79, Finley crédite Protagoras d'avoir été le seul théoricien de la démocratie antique. Aucun texte authentique sur ce sujet n'a survécu. Seul témoigne le Protagoras de Platon ${ }^{13}$ que, sans citation précise, Finley soupçonne être l'objet d'une hargne particulière : "Ses idées (de Protagoras) nous sont connues par les attaques que Platon dirige contre lui dans un de ses premiers dialogues, le Protagoras, dans lequel Socrate utilise moqueries, parodies, voire tricheries, à un degré tout à fait rare dans le corpus platonicien ». Protagoras devrait ce traitement de faveur au fait d'avoir attribué à

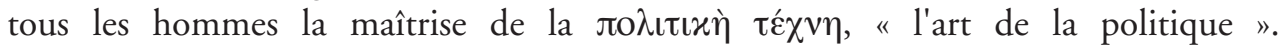
Mais quelles théories peut-on attribuer à Protagoras en l'absence de tout témoignage direct ? La question fait depuis longtemps l'objet de controverses sans fin. Il est vrai que Socrate biaise d'emblée puisque, tandis que Protagoras dit explicitement que son

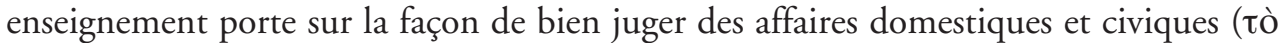

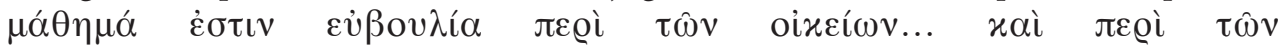

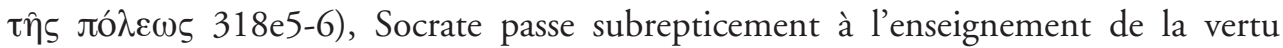

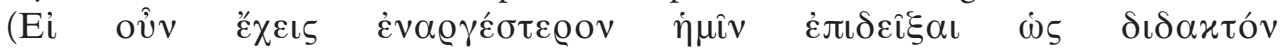

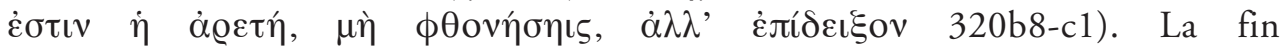
du dialogue est un petit délice de retournement ironique, Socrate prétendant que les thèses se sont inversées, lui-même en venant à penser que la vertu peut s'enseigner, tandis que Protagoras a suivi le chemin inverse, pensant désormais qu'elle ne peut s'enseigner.

Il n'empêche, que, une fois n'est pas coutume, Moses Finley livre une appréciation sur l'écriture du Protagoras et sur les procédés platoniciens dont les contemporains, qui pouvaient disposer des textes authentiques, saisissaient bien mieux que nous, 2500 ans plus tard, la teneur, les raisons et les sous-entendus.

P. 81, pour montrer que l'apathie n'est pas supposée par la législation, Moses Finley cite la loi de Solon, telle que la rapporte Aristote (Constitution d'Athènes 8, 5) : "Quiconque, alors que la cité est en dissension, ne met pas d'arme à disposition ${ }^{14}$ de l'un ou l'autre côté, qu'il soit privé de ses droits et ne participe pas à la vie de la cité. » Cette loi, qu'elle soit authentique ou fictive, n'est pas un texte "littéraire » et n'a pas à être interprétée comme tel. Il n'empêche qu'elle exprime l'obligation pour le citoyen dans une démocratie de prendre parti. De facto l'abstentionniste s'exclut de la communauté civique.

13 En note, Finley exclut, comme il se doit, le Théétète dont le sujet est tout autre : la science (n. 29, p. 176).

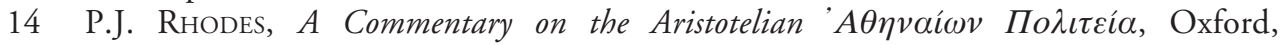
Oxford University Press, 1993, p. 157-158, relève, à la suite de J.A. Goldstein, que

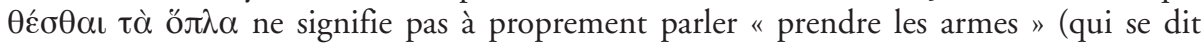

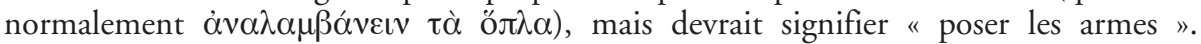
Il suggère le sens de "mettre les armes à disposition ». Je me demande s'il ne faut pas prendre $\mu \eta \delta \varepsilon ́$ au pied de la lettre et comprendre qu'il y a trois possibilités : 1. prendre parti pour une faction, 2. prendre parti pour l'autre, 3. s'armer pour s'ériger en arbitre. 


\section{Chapitre 2. La démocratie, le consensus et l'intérêt national (p. 91-132)}

P. 106, à l'appui de l'idée selon laquelle aucun Grec (Athénien) de l'époque (ve siècle avant notre ère) n'a douté de l'étroit rapport existant entre démocratie et empire, Moses Finley cite une fois encore la Constitution d'Athènes du pseudo-Xénophon : "Ceux qui assurent la marche des vaisseaux sont ceux qui possèdent le pouvoir dans l'État " (pseudo-Xénophon, Constitution d'Athènes 1,2). Le texte est légèrement gauchi. Le pseudo-Xénophon dit exactement : "Je dirai d'abord que l'on croit juste ici que les pauvres et le peuple comptent plus que les nobles et les riches, pour cette raison que c'est le peuple qui mène les navires et qui impartit sa puissance à la cité, que ce soient les pilotes, les donneurs d'ordres, les cinquanteniers, les vigies de proue, les constructeurs de navires, ceux-là sont ceux qui impartissent plus à la cité que les hoplites, les nobles et les gens de bien. Puisqu'il en est ainsi, tous croient qu'il est juste qu'ils participent aux magistratures, par tirage au sort comme par vote et qu'il est permis à qui le veut parmi les citoyens de s'exprimer. " Je me demande si le vieil oligarque n'ironise pas, se donnant pour réaliste bien qu'il tempère son propos de deux " on croit » (ठо Finley, lui, interprète la phrase qu'il a remodelée comme une condamnation. À la suite, il cite une dénonciation de l'avidité du peuple : « Les gens du peuple demandent à être payés pour chanter, courir, danser, naviguer, pour pouvoir ainsi gagner de l'argent et appauvrir les riches "; on peut ajouter la phrase suivante : «Et, dans les tribunaux ils ne se préoccupent pas de la justice plutôt que de leur intérêt " (pseudo-Xénophon, Constitution d'Athènes 1, 13, traduction de Finley-Monique Alexandre). Magnanime, Finley ne juge pas nécessaire de relever le ridicule du propos! Il souligne seulement que ce n'est pas le régime démocratique qui suscite l'empire mais l'inverse : "Notre libelliste ne condamnait pas l'empire, mais bien le système démocratique athénien qui était fondé sur lui. »

P. 106, pour prouver l'absence de justification idéologique de l'Empire, Finley cite, une nouvelle fois, l'oraison funèbre de Périclès (Thucydide, Guerre du Péloponnèse II 41, 3-4) : "Athènes est la seule cité qui règne sur ses sujets sans qu'ils puissent se plaindre de se trouver soumis à une nation indigne d'exercer cette autorité " (traduction de D. Roussel). Qu'en termes cyniques, l'autosatisfaction s'exprime! Pour en goûter le sel, il faut citer l'ensemble de la phrase : "Seule des cités d'aujourd'hui, elle (Athènes) est supérieure à sa réputation, quand elle s'affronte à l'épreuve et seule, elle n'est l'objet ni d'indignation de la part de l'ennemi qui l'a attaquée et a vu à qui il doit la calamité qu'il subit, ni de récrimination de la part de quiconque lui est soumis, sous le prétexte qu'il n'est pas dirigé par des gens qui le méritent " (ma traduction).

P. 107, à la conception idéalisée du pouvoir présentée par Périclès, Finley oppose la brutalité bien connue de Thrasymaque raillant Socrate, telle que la met en scène Platon (République I 343B) : "En politique, le chef authentique regarde ses sujets exactement comme un troupeau et ne pense jour et nuit qu'au profit qu'il peut en tirer 
lui-même. " Comme souvent, la citation est plutôt une paraphrase parce que la lettre du texte, en l'occurrence, n'arrête pas l'attention de Moses Finley. Le propos complet mérite d'être rappelé : "Tu t’imagines que les bergers ou les bouviers visent le bien des moutons ou celui des bœufs, qu'ils les engraissent et les soignent en considération d'autre chose que le bien de leurs maîtres et le leur propre ; en particulier, tu penses que les dirigeants des cités, ceux qui les dirigent vraiment, ont envers ceux qu'ils dirigent d'autres pensées que les dispositions que l'on a envers les moutons et qu'ils visent en eux jour et nuit autre chose que ceci : obtenir eux-mêmes un profit " (ma traduction). Le propos, impudent, se suffit à lui-même.

P. 109, examinant la question de la raison d'État, Finley fait référence au début de la Politique : "Lorsqu'Aristote déclarait (Politique I 1253a19-20) que la polis (la cité-État) est antérieure à l'individu, il entendait cela dans le cadre de sa téléologie : l'homme est par nature un être destiné à vivre dans la polis, la plus haute forme de koinônia, communauté ; c'est la fin ou le but de l'homme, qu'il atteint s'il réalise toutes les potentialités de sa nature. Lorsqu'Aristote jugeait des mérites d'un gouvernement selon qu'il gouvernait ou non dans l'intérêt de la communauté tout entière, ses critères n'avaient rien de commun avec les arguments modernes tirés de la Raison d'État. Il jugeait l'État selon les critères de la justice et de la "vie bonne”. " La phrase bien connue d'Aristote n'est pas citée dans son intégralité : "La cité est par sa nature quelque chose d'antérieur à la maisonnée et à chacun d'entre nous, parce que le tout est nécessairement antérieur à la partie » (Politique I 1253a19-20), mais l'analyse des raisons de l'aphorisme et le commentaire du contexte sont produits. Le postulat selon lequel la cité précède la famille n'est pas et n'a pas à être, dans le contexte, soumis à la critique. Le principe seul en est explicité : selon Aristote, le but du politique relève de l'éthique, puisque c'est " la vie bonne".

P. 116, pour rappeler que "l'explication historique n'est pas identique au jugement moral ", Finley tire, d'une seule phrase, sans référence précise, la conséquence théorique des livres V-VII de la République de Platon (471C-541B) : "Platon était inflexible sur ce point : tous les États, disait-il sans cesse, sont défectueux de manière irrémédiable, l'État juste, l'État idéal sera gouverné par les Rois philosophes grâce à leur appréhension des formes idéales et non à partir d'une étude de sociétés historiques. » On ne saurait mieux construire, en synthèse, une doxa philosophique de facture théophrastéenne!

\section{Chapitre 3. Socrate et après Socrate (p. 133-171)}

P. 136, traitant de l'équilibre délicat dans une société entre l'obéissance et le droit à la parole, Finley ne peut manquer d'évoquer, ne fût-ce qu'en passant, la situation archétypale de Thersite dans l' Iliade (II 211-277) : "Si Thersite fut battu et réduit au silence par Ulysse pour avoir osé proposer aux guerriers assemblés l'abandon de l'expédition troyenne, c'est parce qu'il était un homme du peuple; tout "héros" pouvait 
émettre une proposition avec toute la liberté et tous les dangers que cela comportait du point de vue de l'intérêt commun. "C'est que, dans la société héroïque, les paroles n'ont pas la même légitimité, sinon le même poids, selon que l’on est " héros » ou " homme du peuple ». On peut ajouter que l'intervention de Thersite est précédée, en préambule, d'une action d'Ulysse parcourant les rangs des Achéens, apaisant les rois

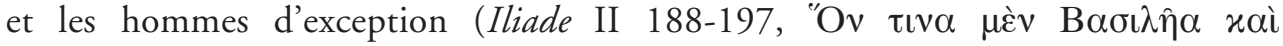

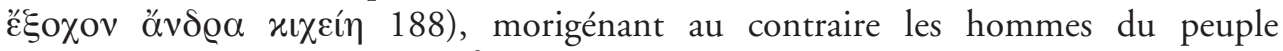

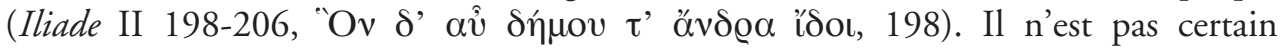
que les institutions de l'ostracisme et de la graphé paranomôn aient réussi (et puissent réussir) à annihiler ou juguler les détenteurs abusifs de la parole et de l'autorité, d'un côté comme de l'autre!

P. 144, Finley rappelle la carrière d'Aristophane, pendant et après la guerre du Péloponnèse, et évoque la scène finale des Acharniens d'Aristophane (425 avant notre ère) : "Dans la scène finale, le vieux paysan [Dicéopolis] qui en est le protagoniste conclut sa propre paix avec l'ennemi, dans un débordement d'absurdité qui n'est pas dénué d'une certaine amertume. "Cette " amertume " est la seule appréciation ponctuelle de psychologie littéraire (plutôt qu'éthique) parmi les citations que fait Finley de textes antiques dans Démocratie antique et Démocratie moderne. Peut-on sérieusement reprocher de dédaigner la littérature à un homme qui résume ainsi le talent d'Aristophane : "Il était d’une gaieté tapageuse, immodérée, scatologique, obscène, railleuse ; il possédait une inépuisable créativité et le don de trouver matière à esprit et humour dans les points faibles des hommes publics, à commencer par Périclès, dans les caractéristiques de l'Athénien moyen, dans les causes de la guerre et dans son déroulement, et même dans les mythes et dans les rites familiers. "

P. 144 aussi, il résume Lysistrata (411 avant notre ère) et la situation critique d'Athènes : "Tout espoir de victoire paraissait désormais reposer sur l'appui financier de l'ennemi traditionnel des Grecs, le Roi de Perse... À un certain niveau, la Comédie est un jeu érotique continu, mais, en transparence, il y a un thème sérieux, assez explicite en deux passages (v. 1124-1135, 1247-1272) : les Perses demeureront les seuls vainqueurs si la guerre se prolonge ${ }^{15}$. " Au passage la qualification " jeu érotique continu » relève elle aussi de l'interprétation littéraire. À la prise de position pacifiste

15 Renvoi est fait, n. 5 p. 181, à Cavaliers 477-478 (424 avant notre ère), Paix 107-108 (421 avant notre ère), Thesmophories 335-8 (411 avant notre ère). Dans sa note 25, p. 180, Finley récuse les interprétations anachroniques et individualistes de K.J. Dover (article "Aristophanes » de l'OCD, $2^{\mathrm{e}}$ éd. 1970 - dans la 4 e édition, l'article a été revu par C.B.R. Pelling, mais l'hypothèse de l'individualisme et l'anachronisme n'ont pas été gommés ; voir aussi de K.J. Dover, Aristophanic Comedy, London/Berkeley, Batsford, 1972), et se réclame de l'analyse des " conceptions politiques d'Aristophane " proposée par G.E.M. DE STe-Croix, The Origins of the Peloponnesian War, London, Duckworth, 1972, ce qui n'est pas sans humour quand on connait leurs controverses ("weberianisme " de Finley contre « marxisme » de De Sainte-Croix). 
qui fait encore le succès de Lysistrata aujourd'hui, Finley ajoute en filigrane l'intention politique : suggérer que le conflit ne profite pas aux belligérants, mais au Grand Roi. Le développement s'inscrit dans un exposé sur la liberté de parole en politique, notamment en temps de guerre. Finley rappelle comment, dès 426, Cléon avait, sans succès, tenté de neutraliser la liberté de parole ( $\alpha \alpha \varrho \varrho \eta \sigma i ́ \alpha)$ d'Aristophane et avait échoué, ne serait-ce que parce que les moqueries faisaient partie d'une "manifestation civique suscitée par l'État sous le patronage d'une divinité, Dionysos ». La religion de la cité autorisait et cautionnait les débordements, non sans toutefois les contrôler.

P. 158, Finley pose que Xénophon (Apologie) et Platon (Apologie, mais en retrait) insistent tous deux sur le rôle de Socrate comme enseignant. Xénophon, particulièrement, paraphrase une apostrophe de Socrate face à Mélétos : "Cite-moi un homme que j'ai corrompu, que j’ai rendu impie de pieux qu'il était". Mélétos réplique : Je peux citer ceux que tu as persuadés de suivre ton autorité plutôt que celle de leurs parents". "Oui, répond Socrate, mais en matière d'éducation, on devrait faire appel à des experts, non à des proches. Vers qui doit-on se tourner, lorsqu'on a besoin d'un médecin, d'un général, vers ses parents ou ses frères, ou vers ceux qui sont les plus qualifiés par leur savoir ?”" (Xénophon, Apologie de Socrate $\$ 19-21$ ). Finley a ici l'art de condenser un texte diffus. Il voit dans cet affrontement l'expression du conflit suscité $\mathrm{au} \mathrm{V}^{\mathrm{e}}$ siècle avant notre ère par la révolution pédagogique, introduite par les Sophistes, au profit des jeunes gens de la classe aisée, Mélétos représentant pour Xénophon le " conservatisme et le traditionalisme aveugle ", en un mot "l'obscurantisme ", comme Anytos dans le Ménon de Platon (p. 160-161) : "Dans l'un de ses dialogues, le Ménon, Platon fait la satire de cette attitude (immobiliste) en mettant en scène Anytos, le plus important des accusateurs de Socrate, comme porte-parole du conservatisme et du traditionalisme aveugle. "Ce ne sont pas les Sophistes qui sont fous, fait dire Platon à Anytos (92A-B), mais plutôt les jeunes gens qui leur donnent de l'argent et encore plus les parents qui leur livrent leurs enfants ; mais plus que tous et de beaucoup, les cités qui les accueillent, quand elles devraient chasser sans exception tout individu qui fait ce métier, citoyen ou étranger" ${ }^{16}$. "Subtilement, Finley n'en remarque pas moins que Platon ne défend nullement la liberté de pensée et de parole, que paradoxalement Socrate représente moins qu'Aristophane. La condamnation de Socrate n'a pas empê-

16 La référence se fait au Ménon, 92a7-c6, dans le contexte de la discussion sur l'enseignement de la vertu : Finley citant littéralement 92a, Monique Alexandre reprend la traduction de Croiset-Bodin (Platon, Ménon, Paris, cuf, $2^{\mathrm{e}}$ éd. 1942). R.W. Sharples (Plato, Meno, $2^{\mathrm{e}}$ éd, Oxford, Oxford University Press, 1991), p. 97 traduit oi toútoıs

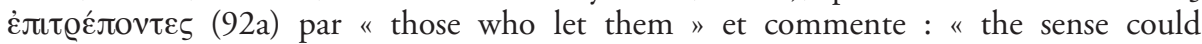
rather be those who hand (the young men) over (to them) sc by failing to intervene ». Il est à craindre que R.W. Sharples, soucieux de déceler quelque laxisme chez les parents,

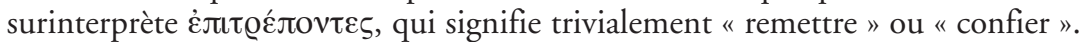


ché Platon d'ouvrir une école dans les jardins d'Académos (vers 387 avant notre ère) ${ }^{17}$ et, à la suite, d'autres maîtres d'ouvrir d'autres écoles. Il est vrai que ce n'était plus des Sophistes itinérants. "Pour Platon, conclut Finley, la condamnation de Socrate symbolisait le caractère pernicieux de toute société ouverte et libre, et pas seulement d'une société démocratique " (p. 163) ; il renvoie finalement au commentaire lapidaire que K. Popper faisait de la peine de mort préconisée pour la récidive (Lois 907D-909D) ${ }^{18}$ : "Platon a trahi Socrate".

P. 161-162, au milieu de l'analyse touchant Platon, s'intercale une évocation d'Aristophane : «Dans les Nuées d'Aristophane, où le "pensoir" de Socrate est incendié dans un finale débridé typiquement aristophanesque, la plupart des traits du portrait de Socrate sont faux, c'est un amalgame des philosophes scientifiques comme Anaxagore, des sophistes et de beaucoup d'invention ; Platon s'éleva là contre avec irritation. » Il est vrai que les bouffonneries aristophanesques frisent l'appel au meurtre (vers 1500, «Tu

17 Sur l'Académie au temps de Platon, voir I. MuelLer, "Mathematical method and philosophical truth, I. Plato's academy and the science ", in R. Kraut (éd.), The Cambridge Companion to Plato, Cambridge, Cambridge University Press, 1992, p. 170-175 ; M.-F. Billot, «Académie (topographie et archéologie) », in R. Goulet (éd.), Dictionnaire des philosophes antiques I, Paris, 1989, Annexe, p. 693-789.

18 L'ensemble du livre X des Lois (884A-910C) traite de l'impiété, le développement sur le châtiment des impies vient, comme il se doit, au terme du dialogue sur le sujet. Platon distingue trois causes d'impiété : 1 . la négation de l'existence des dieux; 2 . le reproche à l'adresse des dieux de ne pas s'inquiéter des hommes ; 3. le reproche fait aux dieux de se laisser corrompre par des offrandes. Chacune des trois causes se divise en deux catégories, selon que les tenants de l'inexistence, de l'indifférence ou de la corruptibilité restent justes ou s'adonnent au plaisir pour le grand dommage $(\lambda \omega \hat{\beta} \beta \eta, 907 \mathrm{C} 5)$ des autres hommes. Tous doivent être emprisonnés en trois sortes de prisons, selon la gravité des charges, l'une

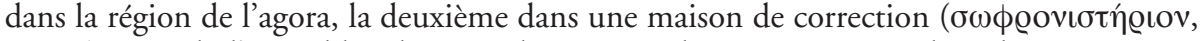
908a5), près de l'assemblée du conseil nocturne, la troisième au milieu du territoire en un lieu désert et sauvage. Les récidivistes seront en effet condamnés à la peine de mort (909a5-8). Ceux qui associeront à l'impiété des pratiques plus que condamnables, telles que la psychagogie, l'incantation, la sorcellerie, seront reclus à vie dans les pires conditions. Platon se délecte à détailler casuistiquement les causes, les motifs, les formes et les sanctions de l'impiété. L'accusation de Mélétos, Anytos et Lycon ne rentre sous aucune des catégories de Platon ou bien, implicitement, sous toutes, puisque, s'il n'honore pas les dieux de la cité, Socrate peut être, au choix, jugé athée, indifférent ou contempteur : "Socrate est dans l'injustice, du fait qu'il ne reconnaît pas les dieux reconnus par la cité et qu'il introduit d'autres divinités nouvelles ; il est aussi dans l'injustice, du fait qu'il corrompt les jeunes gens. » Le plus curieux c'est que l'accusation contre Socrate était le fait d'un trio improbable : Anytos, proche de Théramène, avait d'abord participé à la tyrannie des Trente, mais se montrait farouche partisan de l'accord d'amnistie. De Mélétos II, fils de Mélétos I, on ne connaît que son aspect physique et son goût pour la poésie. Lycon, considéré avec bienveillance par Xénophon dans le Banquet, fut la cible des comiques pour son ivrognerie et son extravagance, Voir D. NaILS, People of Plato, Indianapolis/ Cambridge, Hackett, 2002, s.v. 


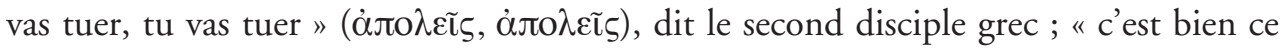

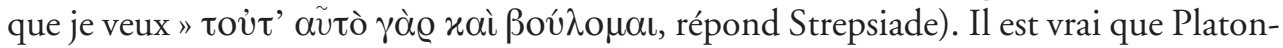
Socrate dans l'Apologie prononce avec férocité un " air de la calomnie " (18a6-20c3, Aristophane est nommément cité en 19c2), il est vrai enfin, si l'on en croit l'autobiographie littéraire de la parabase des Guêpes (v. 1043-1050), que les Nuées essuyèrent à la représentation un cuisant échec ${ }^{19}$. Plus radical, en un sens, Xénophon ne cite nulle part ni même ne nomme Aristophane.

Il serait faux de dire que Finley ne s'intéresse pas à la littérature. Il analyse les textes avec finesse et pertinence. Les exemples relevés ici le prouvent, mais son projet n'est pas tant de scruter minutieusement et philologiquement la lettre des « textes » que de produire des exemples significatifs, sinon familiers, aisément accessibles à quiconque n'est pas novice, et d'en tirer la substantifique moelle utile à sa propre réflexion, en l'occurrence plus orientée vers les démocraties modernes que vers la démocratie antique, qui sert ici d'exemple spéculaire. Souvent Finley substitue à la citation directe une paraphrase ou une exégèse synthétique, ce qui lui permet de résumer en quelques mots jusqu'à deux livres des Lois de Platon ou une comédie d'Aristophane! Le genre de la conférence favorise cette pratique. Les renvois ainsi faits à des textes paradigmatiques n'en sont pas moins suggestifs, invitant l'auditeur ou le lecteur à examiner, à nouveaux frais, les textes proposés. Le philologue lui-même y trouve son compte.

Didier PRALON

Université d'Aix-Marseille

Maison méditerranéenne des sciences de l'homme

5, rue du château de l'horloge BP 647

13094 Aix-en-Provence cedex 2

didier.pralon@wanadoo.fr

19 Aristophane reprit son texte en 418-416 pour une nouvelle représentation, mais n'acheva même pas sa nouvelle mouture (Scholies aux Nuées 552). Le texte que nous lisons aujourd'hui est un composé de l'ancienne et de la nouvelle version. 